\title{
Advancing Healthcare for COVID-19 by Strengthening Providers' Capacity for Best Practices in African, Caribbean and Black Community Service Provision in Ontario: A Multisite Mixed-Method Study Protocol
}

\author{
Josephine Etowa ${ }^{1}$, LaRon Nelson ${ }^{2}$, Egbe Etowa ${ }^{3}$, Getachew Abrha ${ }^{1}$, Janet Kemei ${ }^{1}$, Michelle Lalonde ${ }^{1}$, \\ Jemal Nur ${ }^{4}$, Wale Ajiboye ${ }^{4}$, Ilene Hyman ${ }^{5}$, Sanni Yaya ${ }^{1}$, Hugues Loemba ${ }^{6}$, Robin Taylor ${ }^{7}$, Bagnini Kohoun ${ }^{8}$, \\ Ky'okusinga Kirunga $^{9}$, Onyenyechukwu Nnorom ${ }^{5}$, Sané Dube ${ }^{10}$, Wangari Tharao ${ }^{11}$, \\ Lovelyn Ubangha ${ }^{8} \&$ Bishwajit Ghose ${ }^{1}$ \\ ${ }^{1}$ School of Nursing, Faculty of Health Sciences, University of Ottawa, ON, Canada \\ ${ }^{2}$ Yale school of Public Health, USA \\ ${ }^{3}$ Department of Sociology, Anthropology, and Criminology. University of Windsor, Canada \\ ${ }^{4}$ High Impact Field-Based Interventions (HiFi) Lab, St. Michael's Hospital, Unity Health Toronto, Canada \\ ${ }^{5}$ Dalla Lana School of Public Health, University of Toronto, ON, Canada \\ ${ }^{6}$ Montfort Hospital, Ottawa, ON, Canada \\ ${ }^{7}$ Ottawa Public Health, ON, Canada \\ ${ }^{8}$ Canadians of African Descent Health Organization (CADHO), Ottawa, Canada \\ ${ }^{9}$ African and Caribbean Council on HIV/AIDS in Ontario (ACCHO), Toronto, Canada \\ ${ }^{10}$ Alliance for Healthier Communities, Toronto, Canada \\ ${ }^{11}$ Women's Health in Women's Hands (WHIWH), Community Health Centre, Toronto, Canada
}

Received: March 12, 2021 Accepted: September 20, 2021 Online Published: December 23, 2021

doi:10.5539/gjhs.v14n1p75 URL: https://doi.org/10.5539/gjhs.v14n1p75

\begin{abstract}
Background: The ongoing COVID-19 pandemic has emerged as an unprecedented challenge for public and private life, and healthcare systems worldwide. African, Caribbean, and Black communities (ACB) represent some of the most vulnerable populations in terms of their susceptibility to health hazards, difficulty receiving adequate health care and relatively lower chances of recovery.
\end{abstract}

Objectives: The main aim of this study is to improve the health system's response during and after the COVID-19 pandemic by developing evidence-based models to inform policy and collaborative best practices to mitigate its spread and ameliorate related health consequences in vulnerable communities.

Methods: This is a mixed-method, multisite study based in Ottawa and Toronto that will involve in-depth qualitative interviews and surveys using a structured questionnaire. Data will be analyzed using NVivo for qualitative interviews, Stata 16 and IBM SPSS version 26 for statistical analyses.

Discussion: The findings of this study gained from highly professional health practitioners will produce strong evidence on current gaps in knowledge and practice in the healthcare system's capacity to meet the health needs of ACB population. The distinct insights and perspectives will be disseminated with policymakers and researchers at all levels which will facilitate strategic policy making with the goal of addressing the unique challenges for health promotion among the ACB population that are arising within the COVID-19 context.

Keywords: African, Caribbean, and Black communities (ACB), healthcare system, COVID-19, Ontario

\section{Background}

African, Caribbean and Black (ACB) communities represent some of the most vulnerable populations in Ontario regarding their susceptibility to health risks, difficulty receiving adequate care and lower chances of recovery. For instance, ACB communities account for a quarter of the people living with HIV (PLHIV) in Canada although they constitute merely $5 \%$ of the population (African, Caribbean and Black Communities | The Ontario HIV Treatment 
Network, n.d.). No race-based data is currently being collected on COVID-19 in Canada, but the Black population is overrepresented among hospitalized patients in the US and mortality rates among Black persons ( 92.3 deaths per 100,000 population) are substantially higher than those among white (45.2) or Asian (34.5) persons in NYCi. Similar statistics have emerged in other US cities and states (Osman, 2020).

Social determinants, including structural inequalities and discrimination, account for the disproportionate health risks and differential health outcomes experienced in ACB populations (Kraemer et al., 2020; Wolf et al., 2020; Garrett, 2020; Nicola et al., 2020; Chung, Dong, \& Li, 2020). In the case of COVID-19, excess cases and deaths have been attributed to disproportionately high rates of co-morbid conditions (e.g., diabetes, hypertension) in these communities, as well as structural factors related to income, employment, food insecurity, and the built environment, which necessitate, for example, risky working conditions outside the home and using public transportation. (CBC News, 2020b; Devakumar et al., 2020; Braithwaite \& Warren, 2020; Women on the Front Line (2/3): Cashiers Face "warlike" Conditions Working under Covid-19, 2020; Branigin, 2020; Gawley, 2020; Health workers are the frontline soldiers against COVID-19. Let's protect them $\mid$ Africa Renewal, 2020) Moreover, the same population is over-represented in jails and prisons where social distancing is impossible. (Braithwaite \& Warren, 2020) In Canada, data is limited but we know from national surveys that Black Canadians exceed $50 \%$ on economic vulnerability arising from the COVID-19 crisis; $61 \%$ have seen a decrease in their income, $50 \%$ have difficulty meeting their financial obligations and $47 \%$ have are unable to pay their mortgage or rent on time (Home, n.d.). The increased burden of COVID-19 morbidity and mortality among ACB populations translates into greater challenges for healthcare systems and for governments through loss of social capital, productive labur force, and erosion of cultural equity. (McKee \& Stuckler, 2020; Martínez-Martínez \& Rodríguez-Brito, 2020; New study finds higher social capital and trust leads to better COVID-19 response in some U.S. states | Research \& Innovation, n.d.)

Evidence from previous public health disasters show that one size does not fit all in terms of the inadequacy of traditional risk communication systems for vulnerable populations such as ACB community. (Vaughan et Tinker, 2009; Rowel et al., 2012) Ability to understand and respond appropriately to risk information is influenced by underlying socio-ecological factors such as poverty, low health literacy, sociocultural issues and lack of political voice. (James, Hawkins, \& Rowel, 2007; Bouye et al., 2009) Critical health literacy is becoming a cornerstone in these days of COVID-19 where there is excess information and high expectations to integrate this sea of information into personal behavioral actions to promote health (Abel \& McQueen, 2020). It is also well-documented that ACB communities experience multiple and intersecting barriers to accessing appropriate and responsive health services. These include institutional discrimination; poor representation in healthcare leadership, and research and decision-making; lack of awareness of available services; inadequate culturally appropriate services in relevant languages, and lack of culturally competent health professionals.

In response, numerous scholars have identified the need to build capacity, and reduce stigma and paternalism among health providers working with $\mathrm{ACB}$ communities. ${ }^{29-31}$ Health protection relies not only on a well-functioning health system with universal coverage, but also on social inclusion, justice, and solidarity. In the absence of these factors, inequalities are magnified and scapegoating persists, with discrimination remaining long after (Devakumar et al., 2020). There is also widespread acknowledgment that ACB communities and scholars need to be involved in all aspects of prevention, treatment and outreach. As the COVID-19 pandemic continues unabated, disproportionately affecting the lives and livelihoods of ACB communities, there is an urgent need for research and action to mitigate their health risks and strengthen the capacity of healthcare systems (CBC news, 2020a). Three major research gaps were identified: 1) Information on how factors such as socioeconomic vulnerability, co-morbidity, critical health literacy, and discrimination affect healthcare access and outcomes in ACB communities; 2) Information on the types of knowledge and training needed by frontline health workers (and administrators) in the context of service provision to ACB communities; and 3) Information to inform the development of strategies to ensure health equity and mitigate the impact of COVID-19 in ACB communities. Action towards addressing health inequalities will not only help tackle the pandemic, but also develop better resilience and healthcare capacity building during the post-pandemic era.

Main goals and objectives: The main goal of this study is to improve the health system's response to ACB community during and after the COVID-19 pandemic by developing evidence-based models to inform policy and practice to mitigate its spread and related health consequences in vulnerable communities. The Coordinated Global Research Roadmap (A Coordinated Global Research Roadmap, 2020) recognizes the severe strain placed by COVID-19 on clinical services, including the provision of chronic care services, and the need to focus attention on the current and impending health needs of vulnerable populations. Our specific objectives are to:

1) Engage $\mathrm{ACB}$ communities and health provider stakeholders in research and decision-making processes

2) Examine the contextual vulnerability and challenges experienced by ACB communities 
3) Identify the adequacy and non-intended consequences of current health care practices on ACB communities

4) Increase individual, community and organizational capacity and leadership and generate strategies to address COVID-19 related-health outcomes, and

5) Share new knowledge and support its translation into policy and practice models to mitigate the impact of COVID-19 on ACB communities.

It is well-documented that ACB communities experience systematic bias(es) within the healthcare system. As described in the WHO Global Research Roadmap, there is a strong need to prioritize vulnerable population subgroups suffering from stigmatization and/or with other co-morbid conditions.

\section{Conceptual Framework and Project Management Plan}

The underlying theme and analytical approach of the project is based on the Social-Ecological Model (SEM) that was proposed by McLeroy and colleagues in 1988. This model maintains that the determinants of health go far beyond the biological factors and involve many factors functioning as a subsystems that occur at various levels namely individual, interpersonal, institutional, community, and public policy (Table 1). We have chosen the SEM approach to explore the gaps in healthcare system in the context of COVID-19 that is worsening the health situation and access to care among ACB population. This model enables us to identify the gaps, design interventions, and propose implementation strategies by fully acknowledging the complexity of the situation that are causing the challenges for healthcare systems. This model not only allows us to understand how the sociocultural determinants and healthcare system related factors led to health inequities, but also to build policy capacities to address the root-causes at their respective levels.

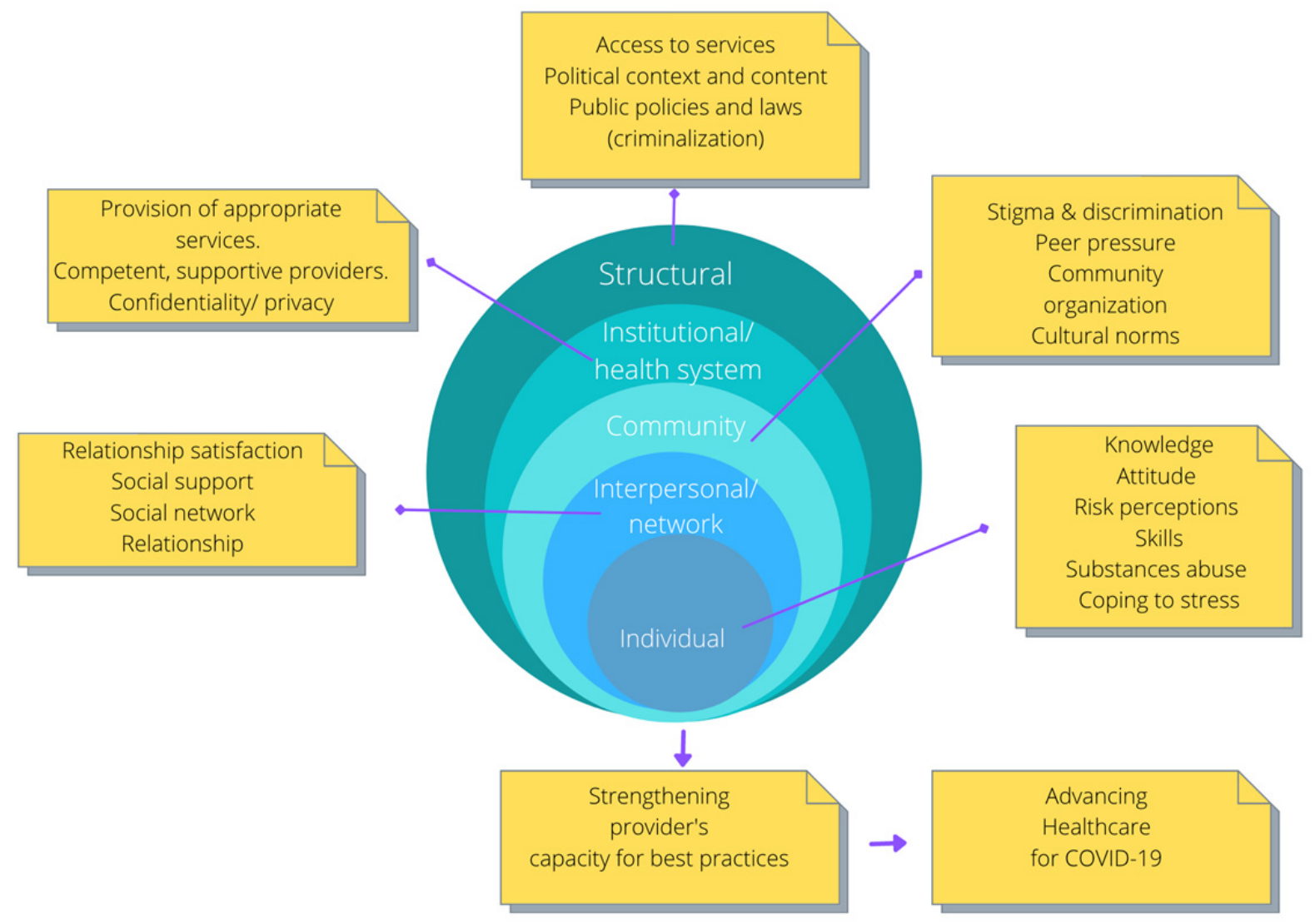

Figure 1. Socioecological Model (SEM) showing the different levels of action to improve COVID-19 related care for racialized communities

Table 1 illustrates the problem statement, major aims, interventions and outcomes specific to the level of analysis including individual, health practitioners and the healthcare system. The outcomes are also classified by their potential impact at their corresponding levels e.g. lowers vulnerability at individual, better quality of care at practitioner, and post-pandemic resilience at healthcare system level. 
Table 1. Problem specification and expected intervention and outcomes

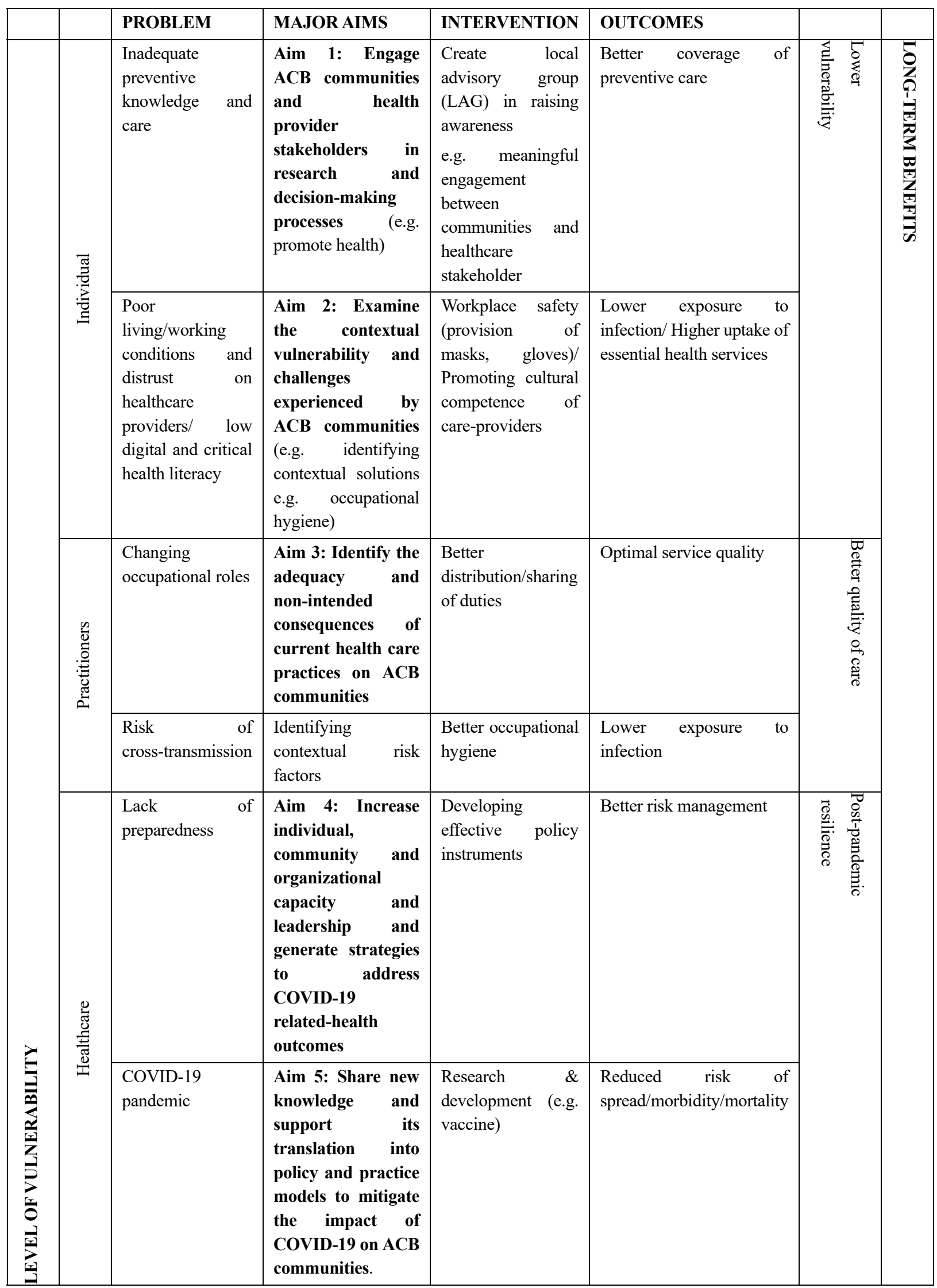




\section{Methodology}

\subsection{Research Design and Data Collection}

In addition to the SEM described above, this mixed-methods research study will be guided by intersectionality (Collins \& Bilge, 2016; Bilge, 2010) and community-based participatory research (CBPR). (Participatory action research (PAR): an approach for improving black women's health in rural and remote communities. - Abstract Europe PMC) Intersectionality framework will help to explicate the vast array of layered macro, meso and micro level factors - i.e., structural, institutional, community, interpersonal and individual - that must be considered when addressing COVID-19 related inequities among racialized communities. CBPR is an approach of working collaboratively with and through groups of people or communities affected by the issues being investigated with a goal of not only studying the issue, but also to address issues affecting the well-being of the study population (Etowa et al., 2018). The project will take place in four (3 months) phases in two sites in Ontario: Toronto and Ottawa.
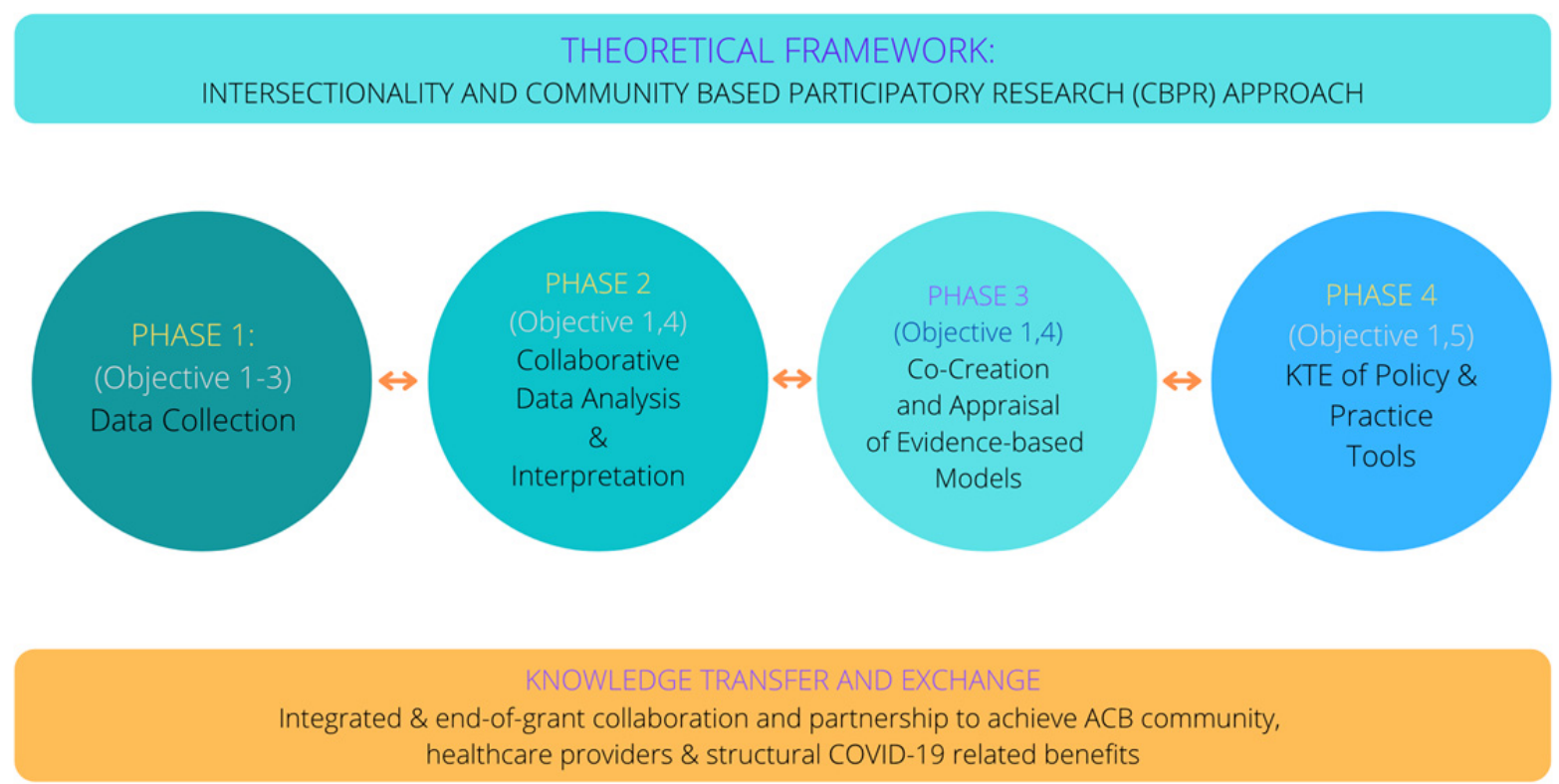

Figure 2. Phases of the project

Phase 1 addresses Aims 1-3 with activities such as creating a local advisory group (LAG), appraising, and synthesizing the growing body of COVID-19 literature, applying for ethical approval, hiring and training research staff, developing research and consent tools, optimizing existing partnerships for collaborative outreach activities, and engaging in recruitment and data collection.

Phase 2 addresses Aims 1,4 and consists of collaborative data analysis and interpretation. Braun and Clarke's six-step framework will inform the thematic analysis of qualitative data (Braun \& Clarke, 2006). We will produce a nuanced analysis of the data in respect of the social determinants of health, our chosen socioecological model, and intersectionality theory. Social identities, primarily gender, but also migration status and SES, that affect risk of COVID-19, health outcomes and experiences with health services, will be integrated into all data collection and analysis to ensure adequate representation.

Phase 3: addresses Aims 1,4 and includes knowledge sharing, appraisal and co-creation of best practice framework using concept mapping strategy. This process will engage ACB community leaders, healthcare providers, policy/decision-makers to develop effective and empowering responses that address vulnerabilities (racism, social and economic marginalization) and promote resilience and critical health literacy in the context of COVID-19, health, and healthcare. Concept mapping has been effectively used to develop a best practices model to address migration and health issues in the ACB community.(Schiller et al., 2013; Haque \& Rosas, 2010) It promotes critical health literacy, which refers to "the degree to which people can access, understand, appraise and communicate information to engage with the demands of different health contexts to promote and maintain good 
health across the life-course. Based on the social distancing orders during this phase, concept mapping may be implemented via face-to-face virtual World café style meetings in both cities with 9-10 focus group discussions (FGDs) in Ottawa and 10-11 in Toronto. FGDs will be transcribed verbatim and thematically analyzed. The scientific rigor of our research qualitative component will be guided by Creswell and Poth (2018) validation strategies. "GroupWisdom" software will be used to perform concept mapping.

The final phase (4) addresses Aims 1, 5, and includes end of grant knowledge exchange activities including the sharing of evidence-based policy tools and practices, and the development of plans to scale up the project. Integrated KT will occur throughout the project and will include a local advisory group which will also serve as a mechanism for consistent community engagement and capacity building (Figure 2).

\subsection{Qualitative Data Collection and Analysis}

The qualitative description approach by Sandelowski will guide the qualitative component of the study. Primary sources of data gathering will be in-depth individual interviews (IDIs) with 100 key stakeholder groups - ACB community $(n=60)$, healthcare providers $(n=30)$ and policymakers $(n=10)$. Focus group discussions (FGD) will also be conducted for data validation through member-checking. Purposive sampling supported by snowball techniques (Creswell \& Poth, 2018) will guide the recruitment of (100) participants comprising of 60 key informants from the ACB community, 30 healthcare providers, and 10 policymakers to participate in this study. Purposeful sampling will allow facilitating the recruitment of participants with rich information, relevant to this study's phenomenon. To ensure participants' representation in both study locations, fifty (50) of these participants will be from the Ottawa area, and fifty (50) will be from the Toronto area. We will use various platforms, including social media, flyers, and referral processes, to recruit participants through our established contacts in the community.

We have developed interview guides for each of the three stakeholder groups; ACB community members, healthcare providers (HCP) and policy makers (PM). group of participants to guide the IDI process. Due to the current COVID-19 restrictions on social distancing, the research assistants will conduct IDIs via Zoom conferencing or any other virtual communication platforms as per the participants' convenience and preference. Each IDI session will last for about 45-60 minutes. Although we have specified the number of participants, past research has indicated that the final sample size often depends on data saturation41-43. Data saturation occurs when further data collection does not reveal new information about the phenomena48. Therefore, we will collect data until data saturation is reached. As per the principles of community-based participatory research frameworks, project advisory committee (PAC) and focus group discussion (FGD) with the stakeholders will also inform the creation of best practice models. 50 Qualitative data analysis will occur concurrently with the data collection. Professional transcriptionists will transcribe data verbatim. Nvivo software will facilitate data management and storage.

Although the numbers of participants have been specified, qualitative research requires data collection until data saturation is achieved in the analysis; when further data collection does not reveal new information. Past research has indicated that the chosen sample size frequently results in data saturation (Morse, 2016; Guest, Bunce, \& Johnson 2006; O'Reilly \& Parker 2012), but the final sample size in our study will be dependent on the point at which saturation is achieved.

Data collection will be conducted in both English and French with targeted efforts via key partner organizations at each site; Ottawa (Montfort hospital, Ottawa Public Health and its affiliates- CHCs, and long-term care facilities) and Toronto (St Mike's Hospital, Taibu CHC; Women's Health in Women's Hands, etc). A number of recruitment strategies will be implemented within these sites, such as distribution of a recruitment email to eligible participants, in-person recruitment through short presentations on site when allowed by Public Health guidelines, as well as distribution of a study flyer via Social Media (such as Facebook, Twitter, and Instagram) through key informants. Potential participants interested in participating in the study are invited to communicate their interest via the study email address.

Braun and Clarke's six-step framework will inform the thematic analysis of qualitative data collected through the in-depth individual interviews and data interpretation to explicate data's commonalities or patterns (Braun \& Clarke, 2006). Nvivo software will facilitate data management and storage. A theme is a pattern in the information that describes and organizes the possible observations, or interpretations of phenomena identified in the data. This process will begin with the development of a coding framework informed by questions from the interview guides and a systematic approach that involves: (1) familiarizing with the data; (2) generating initial codes; (3) developing a coding tree to guide the coding of transcripts; (4) identifying themes; (5) reviewing, defining and naming themes; 6) interpreting the narratives and stories; and (7) producing the report - a concise, coherent, logical, and 
non-repetitive account supported by vivid examples.

Rigour and robustness in qualitative research is to some extent established via a self-conscious and reflexive approach, but it is also accompanied by an explicit methodological framework. To establish the rigour and scientific value of our research, the qualitative component will be assessed with Lincoln \& Guba's criteria, namely, credibility, transferability, confirmability, and dependability. Credibility refers to the confidence observers have in the analytical and interpretive processes and findings. In this research, transparent analytical steps have been identified and informed by established qualitative research principles. Transcripts will be carefully verified and checked. In the analysis process, attention will be paid to misrepresentation of the data. We will interrogate the data until data saturation is reached. Furthermore, the research team is trained in the conduct of ethical and sensitive research with racialized and disadvantaged populations.

Table 2. Qualitative Sampling Techniques and Procedures

\begin{tabular}{|c|c|c|c|}
\hline Study sites & Participant group & Subgroups & Number per site \\
\hline \multirow{6}{*}{ 苞 } & \multirow{2}{*}{ ACB } & Youth & $6\left(3 \mathrm{Eng}^{\mathrm{a}}, 3 \mathrm{Fr}^{\mathrm{b}}\right)$ \\
\hline & & Adult & 14 (8 Eng, 6Fr) \\
\hline & \multirow{3}{*}{ Provider } & Montfort Hospital & 5 \\
\hline & & Nursing home & 5 \\
\hline & & $\mathrm{OPH} \& \mathrm{CHCs}$ & 5 \\
\hline & Policy Makers & $\begin{array}{l}\text { Funder Health organization professional association } \\
\text { community leaders }\end{array}$ & 5 \\
\hline \multirow{7}{*}{ 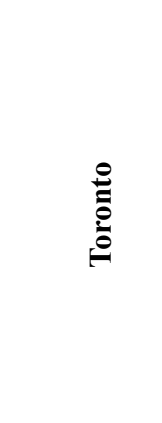 } & \multirow{2}{*}{ ACB } & Youth & 10 (10 Eng) \\
\hline & & Adult & 20 (20 Eng) \\
\hline & \multirow{4}{*}{ Provider } & St Michael Hospital St \& Taibu CHC & 8 \\
\hline & & Nursing home & 3 \\
\hline & & ТPH & 2 \\
\hline & & WHIWH CHC & 2 \\
\hline & Policy Makers & $\begin{array}{l}\text { Funder S, Health organizations, professional associations, } \\
\text { faith-based leaders, community leaders }\end{array}$ & 5 \\
\hline
\end{tabular}

a: Participants whose identified language is English.

b: Participants whose identified language is French.

\subsection{Quantitative Data Collection and Analysis}

Purposive Sampling Technique will be employed to select 1000 Health care providers who are at the heart of COVID 19 fighting's Toronto and Ottawa towns from Ontario in which high number of ACB peoples resides in both towns (Figure 2 below). Stratified Sampling Technique will be used to highlight a specific stratum (Physicians, Respiratory Therapists, Nurse, Personal Support Workers and others.). Final study participants will then be selected from each stratum using Proportional Allocation Sampling Method from health care providers in each stratum. (https://explorable.com/stratified-sampling). 


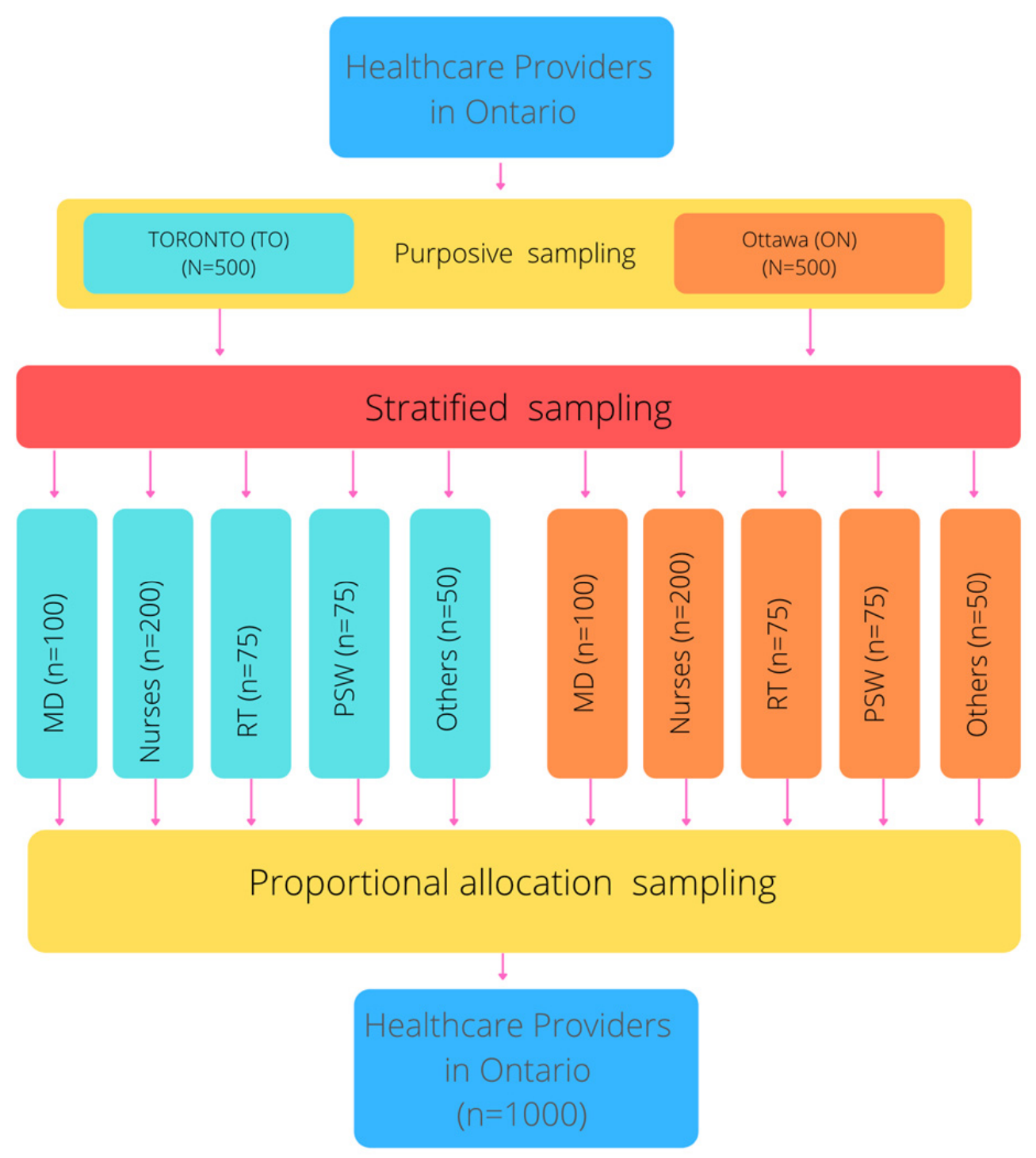

Figure 2. Survey Sampling Techniques and Procedures

Quantitative approaches (1000 online surveys) will be primarily used to collect data from health providers about their knowledge and experience working with $\mathrm{ACB}$ communities and their capacity to respond to the needs of ACB patients during and post-COVID-19. Purposive, stratified, and proportional allocation sampling techniques will be employed. Quantitative data collection will be carried out using Survey monkey. Data collection will be conducted in both English and French with targeted efforts via key partner organizations at each site; Ottawa (Montfort hospital, Ottawa Public Health and its affiliates- CHCs, and long-term care facilities) and Toronto (St Mike's Hospital, Taibu CHC; Women's Health in Women's Hands, etc).

\section{Expected Results/Impacts of the Project}

Both short-term and long-term outcomes and impacts are anticipated. A knowledge translation plan will be developed to ensure that our project findings will be translated into policy and practice. Our main KT goals are to inform health system and facilitate changes in policy and practice to address an immediate response and longer-term consequences of the COVID-19 pandemic. Integrative knowledge translation (IKT) will be implemented within the entire research process. First, we will engage a local advisory group consisting of ACB community members, service providers, knowledge users and project team members to serve as a mechanism for consistent community engagement and capacity building. End of project KT will include the following strategies: 1) We will develop a webinar to share our findings with health and community stakeholders throughout the province. 2) We will host a one-day (virtual) world café style deliberative dialogue/multi-sector think tank with 
6-8 break-out FGDs. This event will engage approximately 100 people, including policy-makers and stakeholders from community and health agencies, ACB service organizations, health professional associations, education, and service users. The goal of this event is to communicate best practices to strengthen the health system's capacity to care for vulnerable populations such as the ACB community. The meeting will begin with a plenary session by presenting the objectives followed by a Q\&A period with the attendees regarding current gaps in meeting the healthcare needs of ACB communities. 3) We will communicate findings to the wider scientific community through presentations at professional meetings and conferences (e.g. CIHR, OPHA, ACCHO, CNA, CPHA Annual Conferences, and poster on partner websites. 4) We will prepare linguistically- and culturally appropriate factsheets, webcasts, and, podcasts on the project website, to improve critical health literacy and ensure the equitable access to our findings to the community at large.

The widespread impact of COVID-19 demands that previously considered local concerns are now global concerns. Provincial collaborations, such as this one, bring different research perspectives to the issue of COVID-19 and health service provision from the perspective of ACB people in Ontario. This project will promote and strengthen knowledge exchange and links to providers, knowledge users and researchers in Ottawa and Toronto with the potential for scale-up, facilitate exposure to new ideas and approaches and provide opportunities for redesigning and identifying promising models for post-pandemic care informed by critical and diverse perspectives. While there are no quick fixes, sustained gains in providers' COVID-19-related health equity capacity such as the one that would be provided by this project will successfully harness knowledge for providers who can address critical post-pandemic health priorities.

\section{Discussion}

The Coordinated Global Research Roadmap recognizes the severe strain placed by COVID-19 on clinical services, including the provision of chronic care services and the need to focus attention on the current and impending health needs of vulnerable populations. Our specific objectives are to: 1). Engage ACB communities and health provider stakeholders in research and decision-making processes 2). Examine the contextual vulnerability and challenges experienced by ACB communities 3 ). Identify the adequacy and non-intended consequences of current health care practices on ACB communities 4). Increase individual, community and organizational capacity and leadership and generate strategies to address COVID-19 related-health outcomes, and 5). Share new knowledge and support its translation into policy and practice models to mitigate the impact of COVID-19 on ACB communities. It is well-documented that ACB communities experience systematic bias(es) within the healthcare system. As described in WHO Global Research Roadmap, there is a strong need to prioritize vulnerable population subgroups suffering from stigmatization and/or with other co-morbid conditions.

This mixed-methods research study will be guided by intersectionality and community-based participatory research frameworks. The project will take place in four phases in two sites in Ontario: Toronto and Ottawa. Phase 1 consists of data collection using both qualitative and quantitative methodologies. In-depth interviews and focus groups will be used to collect data from ACB community members and leaders on socioeconomic and health vulnerabilities considering COVID-19 and health care experiences. An online survey will be used to collect data from health providers about their knowledge and experience working with ACB communities and their capacity to respond to the needs of ACB patients during and post-COVID-19. Phase 2 consists of collaborative data analysis and interpretation. Phase 3 includes knowledge sharing and co-creation of evidence-based models (i.e., concept mapping) with ACB community and health provider stakeholders that will be used to inform policy and practice. The final phase includes the end of grant knowledge exchange activities and the development of plans to scale up the project. Integrated KT will occur throughout the project and will include a local advisory group which will also serve as a mechanism for consistent community engagement and capacity building.

We have strategically assembled a strong team of influential and committed researchers, community leaders, healthcare providers and knowledge users from diverse backgrounds and disciplines across Ontario. They include people at various stages of their career with a track record of collaboration and extensive networks with frontline healthcare providers at the various levels of the system including long-term care facilities where COVID-19 is most felt.

The widespread impact of COVID-19 demands that previously considered local concerns are now global concerns. Provincial collaborations, such as this one, bring different research perspectives to the issue of COVID-19 and health service provision from the perspective of ACB people in Ontario. This project will promote and strengthen knowledge exchange and links to providers, knowledge users and researchers in Ottawa and Toronto with the potential for scale-up, facilitate exposure to new ideas and approaches and provide opportunities for redesigning and identifying promising models for post-pandemic care informed by critical and diverse perspectives. While 
there are no quick fixes, sustained gains in providers' COVID-19-related health equity capacity such as the one that would be provided by this project will successfully harness knowledge for providers who can address critical post-pandemic health priorities.

\section{Conclusions}

The expected outcomes of the proposed research program include: strengthening multisectoral collaborative partnerships in COVID-19 responses of ACB communities; identifying best evidence based models and interventions to strengthen health system's capacity to care for vulnerable populations such as the ACB community, Improving critical health literacy among ACB people and their responses to COVID-19; and generating new knowledge to reduce COVID-19-related health inequities in ACB communities to address the pandemic in Ontario and beyond.

\section{Funding}

This project is funded by Canadian Institute of Health Research (FRN 172739).

\section{Ethical Approval}

University of Ottawa (Supplement file) REB: H-07-20-5984.

\section{Competing Interests Statement}

The authors declare that there are no competing or potential conflicts of interest.

\section{References}

A Coordinated Global Research Roadmap. (2020). Retrieved from https://www.who.int/who-documents-detail/a-coordinated-global-research-roadmap

Abel, T., \& McQueen, D. (2020). Critical health literacy and the COVID-19 crisis. Health promotion international, 35(6), 1612-1613. https://doi.org/10.1093/heapro/daaa040

African, Caribbean and Black Communities | The Ontario HIV Treatment Network. (n.d.). Retrieved from https://www.ohtn.on.ca/research-portals/priority-populations/african-caribbean-and-black-communities/

Baidoobonso, S., Bauer, G. R., Speechley, K. N., \& Lawson, E. (2013). HIV risk perception and distribution of HIV risk among African, Caribbean and other Black people in a Canadian city: mixed methods results from the BLACCH study. BMC public health, 13(1), 1-17. https://doi.org/10.1186/1471-2458-13-184

Bilge, S. (2010). Recent feminist outlooks on intersectionality. Diogenes, 57(1), 58-72. https://doi.org/10.1177/0392192110374245

Bouye, K., Truman, B. I., Hutchins, S., Richard, R., Brown, C., Guillory, J. A., \& Rashid, J. (2009). Pandemic influenza preparedness and response among public-housing residents, single-parent families, and low-income populations. American journal of public health, 99(S2), S287-S293. https://doi.org/10.2105/AJPH.2009.165134

Braithwaite, R., \& Warren, R. (2020). The African American petri dish. Journal of Health Care for the Poor and Underserved, 31(2), 491-502.

Branigin, A. (2020). Black communities are on the "Frontline" of the COVID-19 pandemic: here's why. The Root. Retrieved from https://www.theroot.com/black-communities-are-on-the-frontline-of-the-covid-191842404824

Braun, V., \& Clarke, V. (2006). Using thematic analysis in psychology. Qualitative research in psychology, 3(2), 77-101. https://doi.org/10.1191/1478088706qp063oa

Campbell, K., \& Jones, A. (2009). Prevention Programs in Developed Countries: Lessons Learned: a Report on Prevention Initiatives Used to Address HIV and AIDS Prevention for African, Caribbean and Black Populations in Developed Countries. Interagency Coalition on AIDS and Development. Retrieved from $\mathrm{http} / / / \mathrm{www}$.icad-cisd.com/publication/prevention-programs-in-developed-countries-lessons-learned-a-report -on-prevention-initiatives-used-to-address-hiv-and-aids-prevention-for-african-caribbean-and-black-populat ions-in-developed-count/.

CBC News. (2020a). U.S. Records Deadliest Day yet in Coronavirus Crisis $\mid$ CBC News. CBC. Retrieved April 7, 2020, from https://www.cbc.ca/news/world/us-coronavirus-tuesday-ny-increase-1.5524669

CBC News. (2020b). Money's Not That Important: African Americans in Georgia Wary of Returning to Work as COVID-19 Takes a Toll | CBC News. CBC. Retrieved May 2, 2020 from 
https://www.cbc.ca/news/world/georgia-covid-african-american-1.5552910

Chung, R. Y. N., Dong, D., \& Li, M. M. (2020). Socioeconomic gradient in health and the covid-19 outbreak. Bmj, 369. https://doi.org/10.1136/bmj.m1329

Collins, P. H., \& Bilge, S. (2020). Intersectionality. John Wiley \& Sons.

Creswell, J. W., \& Poth, C. N. (2018). Qualitative inquiry and research design: Choosing among five approaches (3rd ed.). Los Angeles: SAGE Publications.

Devakumar, D., Shannon, G., Bhopal, S. S., \& Abubakar, I. (2020). Racism and discrimination in COVID-19 responses. The Lancet, 395(10231), 1194. https://doi.org/10.1016/S0140-6736(20)30792-3

Djiadeu, P., Nguemo, J., Mukandoli, C., Odhiambo, A. J., Lightfoot, D., Mbuagbaw, L., \& Nelson, L. E. (2019). Barriers to HIV care among Francophone African, Caribbean and Black immigrant people living with HIV in Canada: a protocol for a scoping systematic review. BMJ open, 9(1), e027440. https://doi.org/10.1136/bmjopen-2018-027440

Etowa, J. B., Bernard, W. T., Oyinsan, B., \& Clow, B. (2007). Participatory action research (PAR): An approach for improving black women's health in rural and remote communities. Journal of Transcultural Nursing, 18(4), 349-357. Retrieved from https://europepmc.org/article/med/17911575

Etowa, J., Lisa, P.-D., Adele, V., \& Brianna, K. (2015). Mentoring as a Resource for Aboriginal Nurses: Perspectives of Indigenous Knowers. The International Journal of Organizational Diversity, 14(4), 11-27. https://doi.org/10.18848/2328-6261/CGP/v14i04/40209

Etowa, J., MacDonald, S., Hannan, J., Phillips, J. C., Boadu, N. Y., \& Babatunde, S. (2018). Sociocultural factors influencing infant-feeding choices among African immigrant women living with HIV: a synthesis of the literature. Journal of the American Association of Nurse Practitioners, 30(4), 208-235. https://doi.org/10.1097/JXX.0000000000000014

Garrett, L. (2020). COVID-19: the medium is the message. The lancet, 395(10228), 942-943. https://doi.org/10.1016/S0140-6736(20)30600-0

Gawley, K. (2020). Canada's 'colourblind'coronavirus data could leave officials blind to racial inequities. City News.

Retrieved

from https://www.citynews1130.com/2020/04/07/canadas-colourblind-coronavirus-data-could-leave-officials-bli nd-to-racial-inequities/

George, C., Adam, B. D., Read, S. E., Husbands, W. C., Remis, R. S., Makoroka, L., \& Rourke, S. B. (2012). The MaBwana Black men's study: community and belonging in the lives of African, Caribbean and other Black gay men in Toronto. Culture, health \& sexuality, 14(5), 549-562. https://doi.org/10.1080/13691058.2012.674158

Guest, G., Bunce, A., \& Johnson, L. (2006). How many interviews are enough? An experiment with data saturation and variability. Field methods, 18(1), 59-82. https://doi.org/10.1177/1525822X05279903

Haque, N., \& Rosas, S. (2010). Concept mapping of photovoices: Sequencing and integrating methods to understand immigrants' perceptions of neighborhood influences on health. Family \& Community Health, 33(3), 193-206. https://doi.org/10.1097/FCH.0b013e3181e4bbf0

Health workers are the frontline soldiers against COVID-19. Let's protect them | Africa Renewal. (2020). Retrieved

https://www.un.org/africarenewal/web-features/coronavirus/health-workers-are-frontline-soldiers-against-co vid-19-let\%E2\%80\%99s-protect-them

Home. (n.d.). Association for Canadian Studies. Retrevied from https://acs-aec.ca/en/main.

James, X., Hawkins, A., \& Rowel, R. (2007). An assessment of the cultural appropriateness of emergency preparedness communication for low income minorities. Journal of Homeland Security and Emergency Management, 4(3).

Kraemer, M. U., Yang, C. H., Gutierrez, B., Wu, C. H., Klein, B., Pigott, D. M., ... \& Scarpino, S. V. (2020). The effect of human mobility and control measures on the COVID-19 epidemic in China. Science, 368(6490), 493-497. https://doi.org/10.1126/science.abb4218

Martínez-Martínez, O. A., \& Rodríguez-Brito, A. (2020). Vulnerability in health and social capital: a qualitative analysis by levels of marginalization in Mexico. International journal for equity in health, 19(1), 1-10. 
https://doi.org/10.1186/s12939-020-1138-4

McKee, M., \& Stuckler, D. (2020). If the world fails to protect the economy, COVID-19 will damage health not just now but also in the future. Nature Medicine, 26(5), 640-642. https://doi.org/10.1038/s41591-020-0863-y

Mkandawire, P., Arku, G., Luginaah, I., \& Etowa, J. (2019). Informal transit, socio-spatial exclusion, and changing geographies of HIV/AIDS in urban Malawi. African Journal of AIDS Research, 18(1), 81-88. https://doi.org/10.2989/16085906.2019.1575884

Morse, J. M. (2016). Determining sample size: Qualitative Health Research, juillet. https://doi.org/10.1177/104973200129118183

New study finds higher social capital and trust leads to better COVID-19 response in some U.S. states $\mid$ Research \& Innovation. (n.d.). Retrieved May 3, 2020, from https://research.info.yorku.ca/2020/04/new-study-finds-higher-social-capital-and-trust-leads-to-better-covid -19-response-in-some-u-s-states/

Nguemo, J. D., Iroanyah, N., Husbands, W., Nelson, L. E., Maina, G., Njoroge, I., ... \& Wong, J. (2019). Substance use disorders among African, Caribbean and Black (ACB) people in Canada: a scoping review protocol. $B M J$ open, 9(7), e028985. https://doi.org/10.1136/bmjopen-2019-028985

Nicole, M., Alsafi, Z., Sohrabi, C., Kerwan, A., Al-Jabir, A., Losifidis, C., ... \& Agha, R. (2020). The socio-economic implications of the coronavirus and covid-19 pandemic: A review. International Journal of Surgery, 78, 185-193. https://doi.org/10.1016/j.ijsu.2020.04.018

O'reilly, M., \& Parker, N. (2013). 'Unsatisfactory Saturation': a critical exploration of the notion of saturated sample sizes in qualitative research. Qualitative research, 13(2), 190-197. https://doi.org/10.1177/1468794112446106

Osman, L. (2020). Demographic Data "essential" to Track Spread of COVID-19: Researchers. Rettrieved from https://www.ctvnews.ca/health/coronavirus/demographic-data-essential-to-track-spread-of-covid-19-researc hers- 1.4888879

Rowel, R., Sheikhattari, P., Barber, T. M., \& Evans-Holland, M. (2012). Introduction of a guide to enhance risk communication among low-income and minority populations: a grassroots community engagement approach. Health promotion practice, 13(1), 124-132. https://doi.org/10.1177/1524839910390312

Schiller, C., Winters, M., Hanson, H. M., \& Ashe, M. C. (2013). A framework for stakeholder identification in concept mapping and health research: a novel process and its application to older adult mobility and the built environment. BMC public health, 13(1), 1-9. https://doi.org/10.1186/1471-2458-13-428

Shimeles, H., Husbands, W., Tharao, W., Adrien, A., \& Pierre-Pierre, V. (2011). African, Caribbean and Black Communities in Canada. For the Canadian Institute of Health Research, 1-10.

Social-Ecological Model for Understanding Differential Impact of COVID-19. (n.d.). Retrieved November 18, 2020 ,

from https://curriculum.covidstudentresponse.org/module-3-disparities-policy-socioeconomic-effects/social-ecol ogical-model-for-understanding-differential-impact-of-covid-19

Transformation Through Collective Action: Best Practices in Migration, HIV and Mental Health. (n.d.) ResearchGate. $\quad$ Retrieved May 2, 2020, from https://www.researchgate.net/publication/320665883_Transformation_Through_Collective_Action_Best_Pr actices_in_Migration_HIV_and_Mental_Health

Vaughan, E., \& Tinker, T. (2009). Effective health risk communication about pandemic influenza for vulnerable $\begin{array}{llll}\text { populations. American journal of public health, 99(S2), S324-S332. } & \text {. }\end{array}$ https://doi.org/10.2105/AJPH.2009.162537

Wolf, M. S., Serper, M., Opsasnick, L., O'Conor, R. M., Curtis, L., Benavente, J. Y., ... \& Bailey, S. C. (2020). Awareness, attitudes, and actions related to COVID-19 among adults with chronic conditions at the onset of the US outbreak: a cross-sectional survey. Annals of internal medicine, 173(2), 100-109. https://doi.org/10.7326/M20-1239

Women on the Front Line (2/3): Cashiers Face "warlike" Conditions Working under Covid-19. (2020). France 24. Retrieved April 29, from https://www.france24.com/en/20200429-women-on-the-frontline-2-3-cashiers-face-warlike-conditions-wor king-under-covid-19 
Zhabokritsky, A., Nelson, L. E., Tharao, W., Husbands, W., Sa, T., Zhang, N., ... \& Kaul, R. (2019). Barriers to HIV pre-exposure prophylaxis among African, Caribbean and Black men in Toronto, Canada. Plos one, 14(3), e0213740. https://doi.org/10.1371/journal.pone.0213740

\section{Copyrights}

Copyright for this article is retained by the author(s), with first publication rights granted to the journal.

This is an open-access article distributed under the terms and conditions of the Creative Commons Attribution license (http://creativecommons.org/licenses/by/4.0/). 